\title{
Residual dissection of the brachiocephalic arteries: Significance, management, and long-term outcome
}

\author{
Eugenio Neri, MD \\ Guido Sani, MD \\ Massimo Massetti, MD \\ Giacomo Frati, MD ${ }^{\mathrm{d}}$ \\ Dimitrios Buklas, $\mathrm{MD}^{\mathrm{c}}$ \\ Rossana Tassi, MD \\ Michele Giubbolini, $\mathrm{MD}^{\mathrm{a}}$ \\ Antonio Benvenuti, MD \\ Carlo Sassi, MDa
}

From Dipartimento di Chirurgia, ${ }^{a}$ Unita' Operative di Chirurgia dell' Aorta Toracica e Chirurgia Vascolare, Universita' agli Studi di Siena, Siena, Italy; Istituto di Cardiochirurgia, ${ }^{\text {b }}$ Università degli Studi di Firenze, Firenze, Italy; Service de Chirurgie Thoracique et Cardiovasculaire, ${ }^{\mathrm{c}} \mathrm{CHU}$ de Caen, Caen, France; Istituto di Cardiochirurgia, ${ }^{\mathrm{d}}$ Università "Campus Biomedico" di Roma, Rome, Italy; and Dipartimento di Scienze Neurologiche, ${ }^{\mathrm{e}}$ Università agli Studi di Siena, Siena, Italy.

Received for publication Oct 15, 2003; revisions received Feb 9, 2004; accepted for publication Feb 12, 2004

Address for reprints: Eugenio Neri, MD, Dipartimento di Chirurgia, Universita' agli Studi di Siena, Policlinico le Scotte, Viale M.Bracci, 53100 Siena, Italy (E-mail: euxneri@tin.it nerie@unisi.it).

J Thorac Cardiovasc Surg 2004;128:303-12 $0022-5223 / \$ 30.00$

Copyright (C) 2004 by The American Association for Thoracic Surgery

doi:10.1016/j.jtcvs.2004.02.030
Objectives: Residual dissection of the brachiocephalic arteries after operations for acute type A dissection is considered a benign condition that does not expose patients to late neurologic events. This retrospective study, conducted on an outpatient clinic basis between June 1995 and May 2003, had the objectives of evaluating the consequences of residual dissection of the brachiocephalic arteries, investigating the long-term outcomes of patients with this condition, and illustrating our approach to the condition.

Methods: Forty-two of 137 patients with spontaneous aortic dissection were identified as having residual dissection of the brachiocephalic arteries. There were 30 men and 12 women, with median age of 64.8 years. Patients were followed for a median time of 3.17 years (25th-75th percentile, 1.43-4.40 years; maximum, 7.5 years). The main outcome was the occurrence of cerebral ischemic events (transient ischemic attack or stroke) or death. The functional consequences of brachiocephalic artery dissection were studied by using duplex scanning and transcranial Doppler ultrasonography.

Results: Twenty-four focal neurologic complications occurred in 13 of 42 patients (incidence, 30.9\%); major strokes occurred in 6 patients, and none were fatal. Minor strokes occurred in 12 patients. In all patients the damaged territory was dependent on a dissected artery. Kaplan-Meier (90-months) freedom from focal neurologic events was $55.7 \%$ (95\% confidence interval, 33.7\%-72.9\%). Mean time of freedom from focal neurologic events was 64.5 months (95\% confidence interval, 53.1-75.9 months). Positive transcranial Doppler monitoring for microembolic signals was $24.1 \%$, and patients with clinical symptoms had higher microembolic signal counts than did those without symptoms $(8.4 / \mathrm{h}$ vs $1.9 / \mathrm{h}, P<.001)$. Reduced cerebrovascular reactivity to hypercapnia, calculated by using the breath-holding index values, was associated with severely impaired brachiocephalic artery perfusion. The multivariable model for predictors of late stroke (minor and major) included the following variables: microembolic signal count ( 1 signal $/ \mathrm{h}$ increase; relative risk, 1.27 [95\% CI, 1.12-1.77]), breath-holding index (0.10 increase; relative risk, 0.91 [95\% CI, 0.87-0.94]), and the presence of at least one carotid axis with a thrombosed false channel (relative risk, 0.82 [95\% CI, 0.64-0.93]). Sixteen operations were performed in 12 patients to relieve residual dissection.

Conclusions: These results suggest an increased risk of ischemic events ipsilateral to the dissected arteries. Strict follow-up and identification of subjects at risk implies the exact knowledge of vessel anatomy and perfusion status. Ultrasonographic transcranial Doppler examination plays an important role in the clinical work-up of these patients. 
I n patients with acute type A aortic dissection, open arch repair with hypothermic circulatory arrest and extensive resections of the diseased aorta has the goal of obtaining the complete elimination of the primary tear, obliterating the false lumen, and promoting event-free survival.

Despite these technical refinements, the presence of a perfused false channel beyond the distal anastomosis is common in patients undergoing operations.

Brachiocephalic arteries (BAs) are not infrequently affected by residual dissection; however, this finding is generally considered a low risk for subsequent neurologic events. ${ }^{1,2}$ This retrospective study was undertaken to investigate the significance of residual dissection of the BAs.

\section{Patients and Methods}

Between June 1995 and May 2003, 137 patients with spontaneous type A aortic dissection were followed at the outpatients clinic of our hospital. This surveillance program included patients undergoing operations either in our institution or in other centers $(30 \%)$.

A number of demographic, clinical, pathologic, and surgical variables were collected by studying the patient's hospital records and radiology files and collateral information provided by other hospitals and physicians. The clinical status and the anatomic characteristics of the dissection at the onset and in the postoperative period, as well as the surgical treatment, complications, and all relevant clinical events related to the dissection and its treatment, were recorded.

Residual dissection was defined as the presence of a false lumen, either perfused or thrombosed, which determined a modification of the profile of the branch.

We identified 2 separate groups of subjects from this population: the study group consisted of 42 patients who had residual dissection of BAs, and the control group consisted of 95 patients without residual BA dissection. Table 1 summarizes their clinical characteristics. In 7 patients, although preoperative data indicated a diagnosis of BA dissection, no residual BA dissection and no residual modifications of the vessel profiles could be demonstrated at the moment of hospital discharge (by means of postoperative computed tomographic $[\mathrm{CT}]$ scanning, carotid duplex scanning, or both). These patients, according to the definition, were excluded from the residual BA dissection study group and included among the control subjects.

Among the 42 patients who had residual dissection of the BAs, 12 of these had been treated in other hospitals. There were 30 men and 12 women, with a median age of 64.8 years (25th-75th percentile, 58.5-68.4 years; range, 45-73.8 years). Twenty-nine patients had hemiarch replacement; 9 patients underwent total arch replacement with the button technique, and 4 underwent total arch replacement with separate reimplantation of the supra-aortic branches. In all cases an open technique of anastomosis was used. Seven patients had a mechanical aortic valve (composite graft, $\mathrm{n}=$ 5 ; isolated aortic valve, $\mathrm{n}=1$; mechanical mitral valve, $\mathrm{n}=1$ ).

In all patients BA dissection was a perioperative event. It was documented (by means of preoperative CT scanning, operative reports, or both) to be present at the exordium of the dissection. Table 2 lists the anatomic distribution of the dissected BAs.
TABLE 1. Clinical characteristics and surgical treatment data

\begin{tabular}{|c|c|c|c|}
\hline & $\begin{array}{c}\text { Residual BA } \\
\text { dissection } \\
(n=42)\end{array}$ & $\begin{array}{l}\text { Control subjects } \\
\quad(\mathrm{n}=95)\end{array}$ & $\begin{array}{c}P \text { value } \\
\text { (2-sided) }\end{array}$ \\
\hline Age (y) & \multicolumn{2}{|c|}{$64.8(58.5-68.4) 67.4(50.6-66.9)$} & \\
\hline Sex, male & $30(71.4 \%)$ & $63(66.3 \%)$ & .55 \\
\hline Marfan syndrome & $3(7.1 \%)$ & $12(12.6 \%)$ & .16 \\
\hline Smoker & $10(23.8 \%)$ & $21(22.1 \%)$ & .82 \\
\hline Diabetes & $3(7.1 \%)$ & $15(15.7 \%)$ & .16 \\
\hline CAD & $6(14.2 \%)$ & $13(13.6 \%)$ & .92 \\
\hline Hypertension & $40(95.2 \%)$ & $90(94.7 \%)$ & .90 \\
\hline Atrial fibrillation & $1(2.3 \%)$ & $12(12.6 \%)$ & .05 \\
\hline Mechanical valve & $7(16.6 \%)$ & $31(32.6 \%)$ & .18 \\
\hline Interatrial communication & 0 & $2(2.1 \%)$ & .96 \\
\hline Oral anticoagulants & $12(28.5 \%)$ & $37(38.9 \%)$ & .24 \\
\hline Aspirin & $15(35.7 \%)$ & $21(22.1 \%)$ & .09 \\
\hline $\begin{array}{l}\text { Preoperative stroke } \\
\text { Operation }\end{array}$ & $36(85.7 \%)$ & $11(11.5 \%)$ & $<.0001$ \\
\hline Open technique & $42(100 \%)$ & $81(85.2 \%)$ & $<.0001$ \\
\hline Hemiarch replacement & $29(69.0 \%)$ & $56(58.9 \%)$ & .26 \\
\hline Arch replacement & $13(30.9 \%)$ & $25(26.3 \%)$ & .57 \\
\hline Glue & $35(83.3 \%)$ & $70(73.6 \%)$ & .21 \\
\hline \multicolumn{4}{|l|}{ Cannulation } \\
\hline Femoral (+ other) & $35(83.3 \%)$ & $45(47.3 \%)$ & $<.0001$ \\
\hline Right-left axillary & $5(11.9 \%)$ & $47(49.4 \%)$ & $<.0001$ \\
\hline Direct carotid & $2(4.7 \%)$ & $3(3.1 \%)$ & .64 \\
\hline Antegrade flow* & $23(54.7 \%)$ & $77(81.5 \%)$ & .0014 \\
\hline
\end{tabular}

$C A D$, Coronary artery disease.

${ }^{*}$ Antegrade switch after graft recannulation or axillary perfusion.

TABLE 2. Anatomic distribution of the dissected supraaortic vessel

\begin{tabular}{|c|c|c|c|c|}
\hline & $\begin{array}{c}\text { Vessels } \\
\text { with } \\
\text { dissection/ } \\
\text { patients }\end{array}$ & $\%$ & $\begin{array}{l}\text { Vessels with } \\
\text { flow } \\
\text { obstruction/ } \\
\text { vessels }\end{array}$ & $\%$ \\
\hline Brachiocephalic trunk & $31 / 42$ & 73.8 & $5 / 31$ & 16.1 \\
\hline Right subclavian artery & $25 / 42$ & 59.5 & $9 / 25$ & 36.0 \\
\hline $\begin{array}{l}\text { Right common carotid } \\
\text { artery }\end{array}$ & $24 / 42$ & 57.1 & $11 / 24$ & 45.8 \\
\hline Right internal carotid artery & $16 / 42$ & 38.1 & $4 / 16$ & 25.0 \\
\hline Left common carotid artery & $26 / 42$ & 61.9 & $9 / 26$ & 36.4 \\
\hline Left internal carotid artery & $15 / 42$ & 35.7 & $4 / 15$ & 26.6 \\
\hline Left subclavian artery & $13 / 42$ & 30.9 & $4 / 13$ & 30.7 \\
\hline
\end{tabular}

\section{Follow-up and Data Collection}

The design of the study is a retrospective analysis of the clinical database of our follow-up program. The follow-up regimen of our outpatient clinic consists of some combination of office visits and instrumental investigations. The approach is problem oriented, and therefore some of these studies were performed in all patients undergoing operations for acute type A aortic dissection, whereas dedicated investigations were reserved for patients with special problems. Standard work-up studies are listed in Table 3 for patients with residual dissection of the BAs. These investigations 
TABLE 3. Follow-up investigation protocol

\begin{tabular}{|c|c|c|c|c|c|}
\hline & $\begin{array}{c}\text { Hospital } \\
\text { discharge }\end{array}$ & $1 \mathrm{mo}$ & $6 \mathrm{mo}$ & $1 \mathrm{y}$ & Every year \\
\hline TTE/TEE & - & - & - & - & - \\
\hline $\begin{array}{l}\text { Thoracic/abdominal aorta CT scan } \\
\text { Special investigations }\end{array}$ & $\bullet$ & & - & - & - \\
\hline $\begin{array}{l}\text { Carotid CT scan } \\
\text { Brain MRI/CT scan }\end{array}$ & $\bullet$ & & & $\bullet$ & $\begin{array}{l}\text { Symptoms } \\
\text { Symptoms }\end{array}$ \\
\hline $\begin{array}{l}\text { Carotid/subclavian/vertebral duplex } \\
\text { ultrasonography }\end{array}$ & $\bullet$ & - & - & $\bullet$ & $\bullet$ \\
\hline Transcranial Doppler ultrasonography & - & - & - & - & - \\
\hline Neurologic assessment & $\bullet$ & $\bullet$ & $\bullet$ & $\bullet$ & $\bullet$ \\
\hline Arteriography* & - & Symptoms & Symptoms & Symptoms & Symptoms \\
\hline
\end{tabular}

TTE, Transthoracic echocardiography; TEE, transesophageal echocardiography; CT, computed tomography; MRI, magnetic resonance imaging. *In patients with impaired renal function, arteriography was scheduled within 3 months from hospital discharge.

were prescribed to obtain maximal information about the cerebral perfusion, vascular anatomy, and brain parenchyma status and to monitor possible modifications with time. Patients undergoing operations in other centers before entering the follow-up program underwent these investigations. In the case of modifications of neurologic symptoms or new neurologic symptoms, carotid Doppler studies and brain CT scans were repeated; other investigations were performed according to the indications of the consulting neurologist.

Follow-up information was obtained from hospital outpatient clinic records and through direct contact with the patient, family, cardiologist, and family physician. These were conducted through the months of April and May 2003, and the closing date for inclusion of events was May 31, 2003.

All patients except one, who was lost to follow-up 5 years after the operation, were traced for the common closing date (May 31, 2003). Information about late clinical events and postoperative controls was obtained in all patients, including those without residual BA dissection.

The following clinical events were studied: death, neurologic events (minor and major), and reoperations (on the aorta, the BAs, or both). Transient ischemic attack (TIA) was defined as a transient $(<24$ hours) focal neurologic deficit attributed to cerebral ischemia. Stroke was defined as a focal neurologic deficit lasting greater than 24 hours and attributed to cerebral infarction.

Follow-up was $97.6 \%$ complete. The median follow-up time was 3.17 years per patient (range, 0.4-7.5 years). For the control group, follow-up was $97.9 \%$ complete (93/95), with a median follow-up time of 3.70 years per patient (range, 0.4-7.9 years). The investigation regimen was followed by almost all patients: the minimal rate of patients that at each time point followed their prescribed investigations was within $92.5 \%$ for the BA dissection group and $87 \%$ for the control group.

\section{Instrumental Investigations}

Digital subtraction angiography was performed in all patients to assess both extracranial and intracranial vascular anatomy and to define the presence and degree of collateral circulation.

The anatomic and functional status of extracranial carotid arteries was further defined by means of color-flow B-mode Doppler ultrasound (SONOS 2500, Hewlett Packard, Phoenix, Ariz, or ATL HDI 5000, Philips Medical Systems, Eindhoven, The Neth- erlands; color-coded duplex ultrasound system with linear 7.5$\mathrm{MHz}$, sector $2.5-\mathrm{MHz}$ probe), according to standardized criteria. ${ }^{3}$ Systolic, diastolic, and mean blood flow velocities were measured in each vessel. The vertebrobasilar system was evaluated, as described by Bartels. ${ }^{4}$ Intracranial vessels were examined as described by Aaslid and colleagues ${ }^{5}$ by using a Multi-Dop X/TCD transcranial Doppler instrument (DWL Elektronische Systeme GmbH, Sipplingen, Germany). The patency and flow direction of the major collateral vessels, namely the ophthalmic, anterior, and posterior communicating arteries, was also evaluated. Cerebral vascular resistance to hypercapnia was evaluated by means of the breath-holding index (BHI). This method is considered effective and reproducible in the study of cerebral hemodynamics, both in normal and pathologic conditions. ${ }^{6,7}$ It represents a valuable index of the capability of adaptive changes in cerebral blood flow, which can be reduced or exhausted in patients with carotid occlusive disease. ${ }^{8}$ Bilateral detection of microembolic signals (MESs) was performed by means of insonation of the middle cerebral arteries with transcranial Doppler (TCD) ultrasonography for 30 minutes. MESs were identified according to established criteria. ${ }^{9}$ Bilateral MES counts were performed also in patients with occluded extracranial carotid arteries. Carotid duplex scans and TCD investigations were performed on the days of clinical follow-up.

Before each TCD examination, a standardized questionnaire was administered to disclose clinically manifest cerebral thromboembolic events (eg, transient focal loss of vision, sensory functions, or motor functions or speech disturbances). In cases of cerebral thromboembolism, results of the cerebral CT scan or magnetic resonance imaging were noted.

Transthoracic echocardiography, transesophageal echocardiography, or both were used to assess the status of the aortic root after repair and to evaluate the competence of the aortic valve, as well as to assess the cardiac function. Embolizing cardiopathies, such as dilated cardiomyopathy, patent foramen ovale, atrial thrombus or myxoma, mitral valve stenosis, prolapse or calcification, mechanical cardiac valves, recent myocardial infarction, left ventricular thrombus, and endocarditis, were carefully searched and evaluated.

Two-dimensional and Doppler echocardiography were performed in the parasternal, apical 4-chamber, and subcostal views with a Hewlett-Packard with a 3.5-MHz transthoracic transducer or a $5-\mathrm{MHz}$ transesophageal transducer in the intensive care unit. 
Quantitative regional cerebral blood flow measurements with radionuclide or magnetic resonance imaging techniques was performed episodically and not as a part of a standard follow-up protocol. Therefore data regarding these investigations are not reported.

\section{Functional Evaluation of BA Dissection}

Functional evaluation of BA dissection was based essentially on ultrasonographic investigations, which completed the morphologic aspects demonstrated by means of digital angiography.

The Doppler ultrasound waveforms were classified into 4 categories: (1) normal; (2) high-resistance obstructive flow with severely diminished systolic flow and absent or low diastolic flow; (3) absent flow; and (4) high-velocity flow with increased internal carotid artery peak systolic velocity of greater than $140 \mathrm{~cm} / \mathrm{s}^{10}$ The definition of vessel flow obstruction used in Table 2 included the latter 3 categories and corresponded to the angiographic findings of severe stenosis or occlusion.

Signs on transcranial insonation of collateral flow across the circle of Willis, low flow in the homolateral (dependent) middle cerebral artery, and compensatory increase of blood flow velocity in the contralateral middle cerebral artery confirmed the functional impairment of the carotid artery.

The reserve of vasodilatatory response to cerebral hypoperfusion was evaluated with $\mathrm{BHI}$, according to findings in the literature. ${ }^{11}$

\section{Statistical Analysis}

Distribution-free exact statistical methods were used to avoid unrealistic functional assumptions. Statistical routines implemented in the StatXact, LogXact (Cytel Software Corp, Cambridge, Mass), and $\mathrm{R}^{12}$ statistical packages were used.

Categoric variables were expressed as percentages, and continuous variables were expressed as the median and 25th-75th percentile. A 2-tailed permutation test was performed to test differences among continuous variables. The Fischer exact test was used to test categoric variables. Survival data were analyzed with standard Kaplan-Meier actuarial techniques for estimation of freedom from stroke or survival probabilities and compared with log-rank tests. Cox proportional hazards regression with stepwise addition of variables was used to determine independent predictors of late neurologic events and death. Probability for entry and removal into the model was set to .05 and .10, respectively. Independent predictor variables that contributed to the final multivariate model were considered significant risk factors for late neurologic events or death if the $P$ value for improvement in $\chi^{2}$ tests was .05 or less. Relative risks (RRs) and 95\% confidence intervals (CIs) were reported to indicate the effect size. The cohort of 95 patients not affected by residual BA dissection was used as a control population for comparisons. These patients were entered, together with those with residual BA dissection, in the multivariable models used to determine independent predictors of late neurologic events and death. In this case the presence of BA residual dissection was considered a dichotomous variable entered in the models.

\section{Results}

\section{Population}

There were no differences between patients with residual dissection of the supra-aortic vessel and the control popu- lation with regard to the majority of demographic and clinical characteristics (Table 1). The higher incidence of preoperative stroke corresponded to a higher prevalence of direct involvement of the cervical arteries and to their different characteristics. In the control group preoperative stroke was attributable to various mechanisms: in addition to common carotid dissection (7 patients), other causes were intimal channel intussusception with BA ostial flap obstruction (1 patient) and compression of the true channel at the level of the transverse aortic arch (3 patients). In the control group common carotid dissection, if present at exordium, was (by definition) followed by the complete postoperative healing of the false lumen. In these patients the extension of the intimal flap was more limited than in patients with residual $\mathrm{BA}$ dissection, and in no case did it extend beyond the proximal one third of the artery. Of note, all 7 of these patients underwent aortic repair with axillary artery cannulation for arterial inflow.

Other relevant differences between the study group and the control subjects regarded the surgical technique. The aortic arch was replaced more frequently in the presence of BA dissection, although the difference with control subjects was not significant. Similarly, there was not a statistical difference between the 2 groups with regard to the use of biologic glue. The perfusion technique differed significantly $(P<.0001)$ : antegrade perfusion, using axillary artery cannulation or direct graft recannulation, was more frequent in patients without residual dissection, whereas among patients with persisting dissection, femoral perfusion was used more frequently.

\section{Anatomy}

Of the 42 patients seen in follow-up, there were 88 BAs involved, and 19 had a thrombosis of the false channel. Dissection entailed a severe stenosis or complete obstruction in $29(32.9 \%)$ of the 88 dissected vessels (12 had a thrombosed false channel).

None of the patients presented with an isolate dissection of the innominate artery without involvement of the right subclavian artery, the right carotid artery, or both.

In $8(19.0 \%)$ patients the common carotid artery was affected bilaterally, and in $5(11.9 \%)$ patients both subclavian arteries were involved by dissection. Furthermore, 12 (28.5\%), 22 (52.3\%), and $8(19.5 \%)$ patients had 3, 2, and 1 vessels, respectively, involved simultaneously by dissection.

Critical flow abnormalities of the carotid arteries were present in 22 patients. Table 2 shows the details of anatomic involvement. In this patient population, the degree of arterial stenosis did not modify spontaneously through the time of the study.

Four patients presented with complete occlusion of the right common carotid artery, and 1 patient presented with 
TABLE 4. Transcranial Doppler findings in patients with carotid dissection

\begin{tabular}{|c|c|c|c|c|}
\hline Variable* & $\begin{array}{c}\text { Group 1: No flow } \\
\text { obstruction } \\
(n=22)\end{array}$ & $\begin{array}{l}\text { Group 2: Flow } \\
\text { obstruction, no } \\
\text { symptoms } \\
\text { (n = 12) }\end{array}$ & $\begin{array}{l}\text { Group 3: Flow } \\
\text { obstruction, } \\
\text { clinical } \\
\text { hypoperfusion } \\
\text { (n= 8) }\end{array}$ & $P$ valuet \\
\hline $\mathrm{MAP}(\mathrm{mm} \mathrm{Hg})$ & $74.1(70.1-81.3)$ & $82.2(73.9-86.7)$ & $87.6(83.2-96.2)$ & .002 \\
\hline $\mathrm{BFV}_{\mathrm{s}}(\mathrm{cm} / \mathrm{s})$ & $117.4(110.5-121.6)$ & $105.7(102.2-127.8)$ & $91.8(86.3-92.9)$ & $<.0001$ \\
\hline $\mathrm{BFV}_{\mathrm{D}}(\mathrm{cm} / \mathrm{s})$ & $58.0(55.7-62.2)$ & $53.8(51.2-57.0)$ & $46.5(43.5-48.9)$ & $<.0001$ \\
\hline $\mathrm{BFV}_{\mathrm{M}}(\mathrm{cm} / \mathrm{s})$ & 76.2 (72.9-82.1) & $70.7(67.1-75.7)$ & $61.0(57.0-63.2)$ & $<.0001$ \\
\hline $\mathrm{BFV}_{\mathrm{S} \text { contra }}(\mathrm{cm} / \mathrm{s})$ & $115.9(110.1-121.2)$ & $118.5(116.6-125.7)$ & $99.2(97.3-111.2)$ & .004 \\
\hline $\mathrm{BFV}_{\mathrm{D} \text { contra }}(\mathrm{cm} / \mathrm{s})$ & $53.8(50.6-56.6)$ & $58.0(55.9-60.8)$ & $46.5(42.1-48.8)$ & .002 \\
\hline $\mathrm{BFV}_{\mathrm{M} \text { contra }}(\mathrm{cm} / \mathrm{s})$ & $70.7(66.7-75.1)$ & $76.2(73.8-80.0)$ & $61.0(58.3-65.7)$ & .001 \\
\hline $\mathrm{BHI}$ & $1.07(0.99-1.18)$ & $0.80(0.56-1.01)$ & $0.37(0.16-0.43)$ & .001 \\
\hline $\mathrm{BHI}_{\text {contra }}$ & $1.12(1.01-1.15)$ & $0.98(0.77-1.13)$ & $0.86(0.48-0.71)$ & .05 \\
\hline
\end{tabular}

The values of middle cerebral artery blood flow velocities and cerebrovascular reactivity to hypercapnia (calculated by using the breath-holding index values in the middle cerebral arteries) are presented.

$M A P$, Mean arterial pressure; $B F V_{S}$, systolic blood flow velocity; $B F V_{D}$, diastolic blood flow velocity; $B F V_{M}$, mean blood flow velocity; contra, contralateral; $B H I$, breath-holding index.

${ }^{*}$ Median (25th-75th percentile).

tBetween the groups.

complete occlusion of the left common carotid artery. The right subclavian artery was occluded in 3 patients, and the left subclavian artery was occluded in 2 patients.

The presence of residual dissection of the BAs in patients who underwent total arch replacement indicates that a more radical operation did not eliminate the false channel. This phenomenon was associated to the distal extension of the dissecting process and to the presence of distal multiple re-entries, which maintained a patent false channel. This occurred in 4 of 13 patients having undergone arch replacement, including the subject who received separate BA reimplantation.

In 2 of these patients, we observed, in the right common carotid axis, a reverse flow in the false channel that was alimented by a re-entry at the level of the carotid bifurcation (or internal carotid artery). The false channel was in large communication at the level of the brachiocephalic trunk, with a dissected right subclavian artery.

In 9 patients arch replacement determined a thrombosis of the false channel that caused a stenosis of the true lumen, and in 6 patients ( 9 arteries) the stenosis was severe.

\section{Cerebral Hemodynamic Status}

The presence of flow obstruction at the level of the dissected supra-aortic vessel did not necessarily imply malperfusion because of the presence of collateral blood flow supply to the brain either through the circle of Willis or the cervical anastomoses between the carotid and subclavian-vertebral systems. ${ }^{13}$ In Table 4 we present the results of TCD measurements undertaken to assess the hemodynamic consequences of BA dissection. We identified 3 groups on the basis of their anatomic and clinical features: patients with carotid dissection but without BA flow obstruction (group 1), patients with carotid dissection with flow obstruction but without symptoms of hypoperfusion (group 2), and patients with carotid dissection with flow obstruction and with symptoms of hypoperfusion (group 3). Flow obstruction refers to the presence of a critical stenosis at any level along the course of the carotid axis, either at the level of the common carotid arteries, the internal carotid arteries, or both.

Significant differences were found between groups that corresponded precisely to the underlying pathophysiologic conditions. In group 3 the finding of a reduced homolateral middle cerebral artery blood flow velocity, together with the failure to ensure adequate collateral flow (weak increase of contralateral flow velocity), explains the onset of symptoms. The profoundly reduced reserve of vasodilatation in patients with symptoms indicates the maximal activation of the compensatory autoregulatory mechanisms.

\section{Neurologic Outcome}

Twenty-six patients experienced neurologic symptoms at the exordium of the dissection. Of these, 15 presented with neurologic sequelae at the moment of the first visit, and 24 had brain CT scan signs of old focal or diffuse damage.

At admission in the follow-up program, 6 (14.3\%) of the 42 patients had recurrent neurologic symptoms attributable to cerebral hypoperfusion; these included dizzy spells, syncope, drop attacks, vertigo, dizziness, or generalized seizures. Another 2 patients became symptomatic later.

During a cumulative follow-up of 49,654 days, 24 focal neurologic complications occurred in 13 of 42 patients (incidence, 30.9\%). Major strokes occurred in 6 patients, 


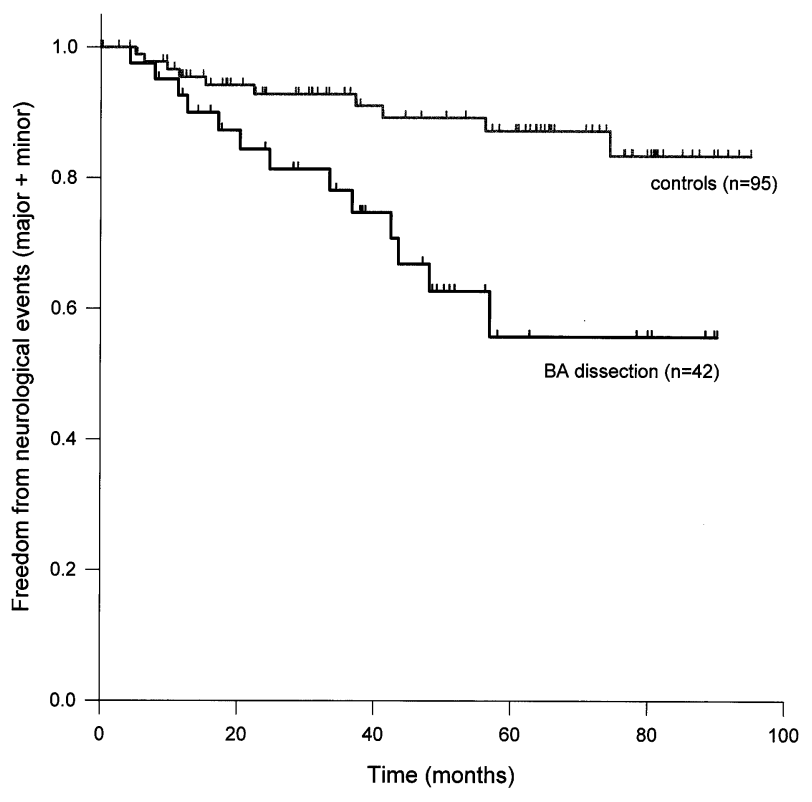

Figure 1. Cumulative rate of freedom from focal neurologic events (major and minor strokes, Kaplan-Meier method) of 42 patients with residual dissection of the BAs and of a control population of 95 patients without BA dissection.

but none were fatal. Minor strokes occurred in 12 patients: in 4 patients ( 7 episodes) they preceded a major stroke, and in 7 patients they were the sole neurologic manifestation. Multiple events (multiple minor events or TIA plus major stroke) occurred in 12 of 13 symptomatic patients. The patients with embolic stroke or TIA had no cardiac source of emboli, as assessed by means of transesophageal echocardiography, and none had mechanical valves. In all patients with a stroke or TIA, the damaged territory was dependent on a dissected artery. None of these patients had primitive, atherosclerotic, carotid, or vertebral artery disease, as assessed by means of angiography or duplex scan studies.

Embolic stroke was diagnosed at CT scanning in 5 patients, whereas a watershed infarction between the territory of the right posterior and right middle cerebral arteries was found in 1 subject. This patient, before the event, had persisting posterior fossa symptoms caused by a stable subclavian steal syndrome and a concomitant dissection of the right internal carotid artery. The event occurred during a documented hypotensive episode in the course of a dialysis session.

As shown in Figure 1, the Kaplan-Meier (90 months) freedom from focal neurologic events (minor and major stroke) was $55.7 \%$ (95\% CI, 33.7\%-72.9\%). The mean time of freedom from focal neurologic events was 64.5 months (95\% CI, 53.1-75.9 months). There was a significant difference from the control population $(P=.0029$ by using the log-rank test).

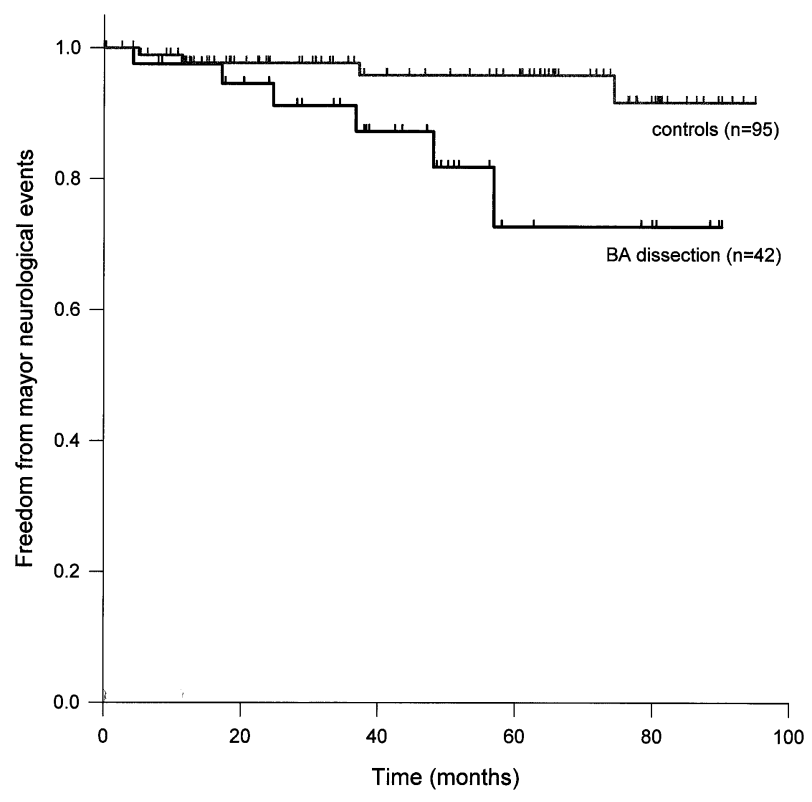

Figure 2. Cumulative rate of freedom from major focal neurologic events (Kaplan-Meier method) of 42 patients with residual dissection of the BAs and of a control population of 95 patients without BA dissection.

In detail, Kaplan-Meier (90-month) freedom from major neurologic events was $72.7 \%$ (95\% CI, 44.5\%-88.2\%), and the mean time of freedom from major neurologic events was 76.1 months (95\% CI, 65.2-87.0 months). There was a significant difference from the control population $(P=$ .0225 by using the log-rank test, Figure 2).

Kaplan-Meier (90-month) freedom from minor neurologic events was $76.6 \%$ (95\% CI, 56.0\%-88.4\%), and mean time of freedom from major neurologic events was 75.5 months (95\% CI, 65.1-85.8 months). There was a significant difference from the control population $(P=.0523$ by using the log-rank test) (Figure 3).

A total of 273 TCD examinations (median, 7; 25th-75th percentile, 4-8; range, 1-11 per patient) were performed. There were $22(52.3 \%)$ patients with at least one positive MES count. Among these, the results of 66 (24.1\%) of 273 examinations were positive, with an MES count median of 3.1/h (25th-75th percentile, 3-7; range, 1-31). Patients with clinical symptoms had higher MES counts than did those without symptoms $(8.4 / \mathrm{h}$ [25th-75th percentile, $6.1-12.4 / \mathrm{h}$ ] vs $1.9 / \mathrm{h}$ [25th-75th percentile, $0.3-4.4 / \mathrm{h}], P<.001$ ).

In the overall population (BA dissection and control groups), the Cox regression RR and 95\% CI for predictors of late stroke (minor and major) were as follows: residual BA dissection, RR of 3.99 (95\% CI, 2.93-11.52); noncontrolled postoperative hypertension, RR of 1.67 (95\% CI, 1.37-7.63).

Within the sample of patients with residual BA dissection, a model that included TCD findings, Cox regression 


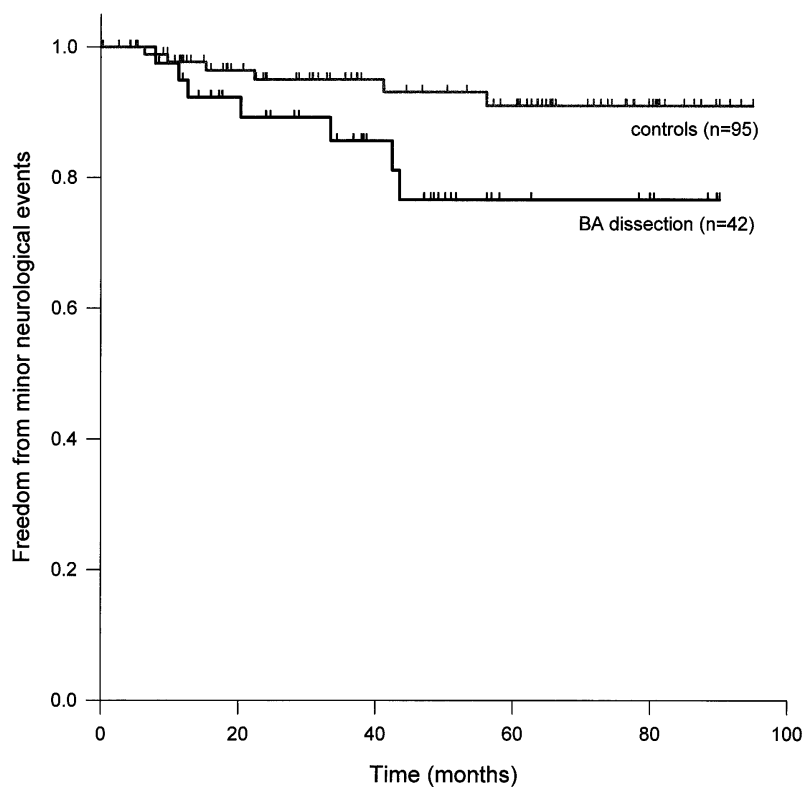

Figure 3. Cumulative rate of freedom from minor focal neurologic events (Kaplan-Meier method) of 42 patients with residual dissection of the BAs and of a control population of 95 patients without BA dissection.

RRs and 95\% CIs for predictors of late stroke (minor and major) were as follows: MES count (1 signal/h increase), RR of 1.27 (95\% CI, 1.12-1.77); BHI (0.10 increase), RR of 0.91 (95\% CI, 0.87-0.94), and presence of at least one carotid axis with a thrombosed false channel, RR of 0.82 (95\% CI, 0.64-0.93). No other variables, such as age, symptomaticity, or middle cerebral artery minimal blood flow velocity (significant at univariate analysis), could be added to improve the model.

\section{Mortality and Morbidity}

None of the patients with major or minor neurologic complications died. Three patients died during the follow-up period: 2 patients experienced fatal aortic rupture and sudden cardiac death after myocardial infarction, respectively, and another patient died of respiratory failure and sepsis after reoperation on the descending aorta and distal arch. These deaths occurred at 214, 363, and 411 days respectively, yielding an overall mortality rate of $7.1 \%(3 / 42)$ for the entire series.

The Kaplan-Meier (90-month) survival rate for the entire series was $92.8 \%$ (95\% CI, 77.3\%-97.4\%), and mean survival time was 83.7 months (95\% CI, 75.4-92.1 months). There was not a different late mortality among patients with residual BA dissection compared with the control population $(P=.16$ by using the log-rank test) (Figure 4$)$.

Cox regression RRs and 95\% CIs for predictors of late mortality were age at the time of the operation (RR, 1.07 per year of age; 95\% CI, 1.03-1.095), renal failure (RR, 1.76;

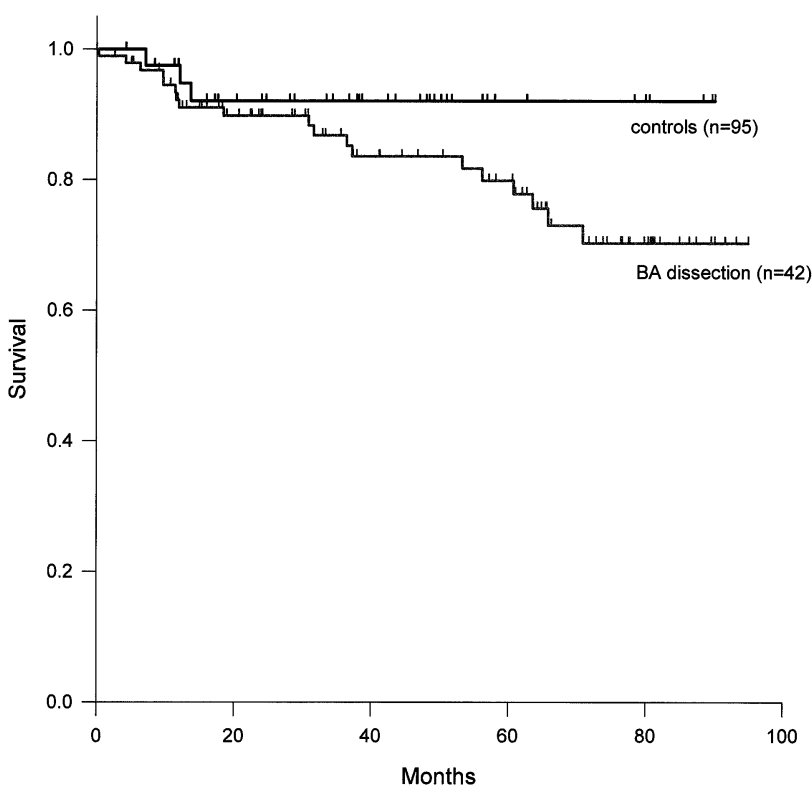

Figure 4. Cumulative survival (Kaplan-Meier method) of 42 patients with residual dissection of the BAs and of a control population of 95 patients without BA dissection.

95\% CI, 1.09-2.86), and congestive cardiac failure (RR, $2.41 ; 95 \%$ CI, 1.62-3.58).

\section{Surgical Treatment}

Secondary operations were performed in 16 of 42 patients with residual BA dissection. We differentiated interventions on the aorta for the aneurysmal evolution of the false channel from those performed to relieve cerebral vessel dissection.

Treatment of the descending aorta for aneurysmal evolution was performed in 4 cases: there were 3 surgical descending aorta replacement procedures and 1 case of thoracic aorta endovascular treatment with a thoracic stent graft.

A total of 12 patients underwent 16 operations for residual BA dissection. The variety of operations performed in this population of patients reflects the assortment of individual problems.

Indications were on the basis of clinical symptoms and of instrumental findings. Eight patients had symptoms of carotid hypoperfusion, 3 patients had symptomatic subclavian steal syndrome, and 2 patients had multiple TIAs despite antithrombotic medications and positive MES counts at TCD examinations.

More radical procedures, with secondary replacement of the transverse arch and individual reimplantation of the brachiocephalic vessels, were performed in 4 patients. Individual BA reimplantation was extended to the neck by using separate prosthetic grafts to relieve compression of the true channel at the level of the common carotid arteries 
(4 left common carotid arteries and 3 right common carotid arteries). These operations, performed during hypothermic circulatory arrest and selective cerebral perfusion, were all done in symptomatic patients. Their median age was 61 years (range, 48-64 years), and the median delay from initial treatment was 17 months (range, 8-25 months). Symptomatic subclavian steal syndrome was treated in 3 patients. Operations performed were 1 left subclavian artery stenting to relieve compression by a thrombosed false channel and 2 carotid-to-subclavian (1 left and 1 right) bypass procedures. In addition, we performed a femoral-to-right-subclavian bypass procedure on a patient who had a complete occlusion of the origin of both the right subclavian and right common carotid arteries caused by the late thrombosis of a brachiocephalic trunk aneurysm (aneurysmal evolution of the false channel). Thrombosis occurred 6 years after the initial operation and determined both hypoperfusion and embolism. Loss of consciousness preceded by focal neurologic signs and epileptic seizures was associated with critical right arm ischemia. The presence of a tracheostomy and the residual dissection of the left subclavian artery recommended this uncommon extra-anatomic bypass revascularization. Righthand amputation became necessary 10 days later.

Endovascular treatment of BA malperfusion was performed in 3 patients ( 6 procedures) in the presence of true lumen compression by a thrombosed false channel: the common carotid artery was treated bilaterally in 2 patients, and in the other patient, right common carotid artery stenting was associated with right internal carotid artery stenting.

Carotid fenestration with surgical removal of the carotid intimal flap was performed in 2 patients. In both cases the compressing false channel determined flow stasis inside the true channel, and a severely diminished peak systolic velocity and a low (or absent in 1 case) diastolic flow (staccato flow) were documented. A similar degree of flow stasis was considered to be the source of emboli (artery-to-artery emboli). Flap fenestration determined the successful decompression of the true lumen, thus resolving the flow stasis, and a synchronous decrease of embolic counts was observed at TCD examination after the operation. None of the patients who underwent secondary operations died or experienced permanent stroke, although temporary neurologic dysfunction with agitation was observed in one patient treated with secondary total arch replacement. Other complications, including atrial fibrillation and bleeding (requiring sternal re-entry), were observed in 2 patients. Neurologic long-term outcome, characterized by no recurrence of symptoms, such as hypoperfusion symptoms, TIA, or strokes, was excellent in all patients.

\section{Medical Regimen}

Medical treatment was not standardized, and the choice of therapy was based on the judgments of the attending physicians. In all patients who experienced focal neurologic events or with positive microemboli counts at TCD, either antiplatelet therapy or anticoagulation was undertaken.

As in all patients with chronic aortic dissection, antihypertensive medications were administered with the objective of reducing both blood pressure and the effect of the slope of the pressure wave. Clinical evaluation of symptoms and TCD findings enabled us to appraise the consequences of pressure adjustments in the presence of BA obstructions or severe stenoses. Resistant hypertension ${ }^{14}$ was quite a common finding in the presence of BA dissection and represented a major therapeutic problem in $9(21.4 \%)$ of 42 subjects. All affected subjects presented with important modifications of the intracranial circulation caused by extracranial vessel flow obstruction.

\section{Discussion}

The present study adds to a small set of clinical articles dealing with persistent dissection of the BAs in patients operated on for type A acute aortic dissection. In 1988, Zurbrugg and colleagues ${ }^{1}$ reported the results of the follow-up (mean follow-up, $43 \pm 38$ months) of 16 patients with residual BA dissection. They found a high recurrence of neurologic events ( 9 patients), but only in 2 cases was there a correspondence with dissected vessels. Also, the average interval from surgical intervention was quite long (44 months), and therefore they concluded that a residual carotid dissection need not be considered a risk factor for subsequent neurologic events.

Zielinski and associates, ${ }^{2}$ in a more recent study, describe the outcome of 15 patients with acute type A aortic dissection who had residual dissection of at least one carotid artery. During a 21-month follow-up, they documented no new major neurologic events, despite the persistence of carotid dissections with different degrees of narrowing of the true lumen.

In the light of these 2 articles, which represent the most recent and extensive contributions on the subject, residual dissection of the BAs appears quite benign and does not impose special precautions during the follow-up. In the light of our findings, we do not share the same optimism.

Although residual BA dissection does not affect longterm survival (Figure 4), patients with this condition are more exposed to neurologic events (TIA and strokes) than patients undergoing operations for acute type A dissection without residual BA dissection (Figures 1-3). Moreover, it was found to be an independent predictor of neurologic events among patients undergoing operations for type A aortic dissection: the RR of 3.99 indicates a 4-fold increase of risk of TIA or stroke in the presence of residual dissection of the BAs.

In our study all events occurred in the territory dependent on a dissected artery, thus underscoring the tight relationship with anatomic disturbances. 
Among patients with residual BA dissection, a multivariable model indicated a role of 3 components in the genesis of focal neurologic disturbances: impaired vasodilatatory reserve, microembolism, and the presence of a perfused false channel.

The fact that the risk of stroke increases about $10 \%$ as BHI decreases by 0.10 gives us a measure of the importance of cerebral autoregulation mechanisms in the genesis of the condition. Reduced BHIs are observed in the presence of reduced middle cerebral artery blood flow velocities, and therefore we suspect that vasodilatatory reserve is impaired because of hypoperfusion and is not an expression of irreversible brain damage. Moreover, this indicates that BA flow disturbances have a role in neurologic events only when they determine severe hemodynamic disarrangements.

Embolic discharge, which is a phenomenon well known to occur in primitive carotid and vertebral artery dissection, ${ }^{15}$ has never been described in patients undergoing operations for acute type A dissection. Although recent literature indicates that most of the MESs occurring in patients with artificial valves are predominantly gaseous and not solid, ${ }^{16}$ in the absence of prosthetic valves, this mechanism, which entails high flow turbulence and cavitation, is still unproved. On the contrary, the flow characteristics observed in some dissected vessels, with phases of stasis and reduced peak velocities (staccato flow), corroborate the hypothesis of artery-to-artery particulate embolism. The successful abolition of MES counts subsequent to the relief of flow stasis in the true channel by means of carotid fenestration further supports this hypothesis and constitutes a potential indication for this operation.

Our data indicate that the source of emboli has little chance of being the false channel. Actually, the presence of a thrombosed false channel in the dissected BAs plays a protective role, reducing by about $18 \%$ (RR, 0.82 ) the risk of neurologic events. Whether the risk of stenosis by the thrombosed false channel would be far less than embolism associated with a perfused false channel is not definitely demonstrated by our data; however, the consequences for initial treatment and for subsequent surgical planning are significant.

Although we have seen that arch replacement did not protect against residual dissection, the majority of patients having undergone this procedure had a thrombosis of the false channel. In the light of our findings, this was a positive issue for stroke prevention.

Still, with regard to surgical management, our data indicate that the use of central cannulation and antegrade arterial flows was associated with a lower risk of residual BA dissection, whereas the use of glue was not.

The presence of a patent residual false lumen after the ascending operation for type A dissection depends on var- ious factors with a reported prevalence of between $30 \%$ and $91 \% .^{17,18}$

Although it has been clearly shown that operative technique and distal level of repair play a major role in reducing this rate, major emphasis has been recently placed on perfusion strategies adopted in occasion of acute-phase operations. ${ }^{17}$ David and coworkers ${ }^{18}$ report a higher prevalence of flow into the false lumen, when patients received retrograde arterial perfusion in comparison with patients who had antegrade arterial perfusion. The anatomic substrate of these findings relies on another study of the same group ${ }^{19}$ that examined the effects of treatment on the characteristics of re-entry tear. They describe the deleterious effects of pressurization of the false channel by the retrograde flow against the aortic clamp, resulting in multiple tears and re-entries.

Although our data do not provide an equivalent level of evidence that the perfusion strategy influences the fate of the residual false channel, we are persuaded that the high prevalence of residual BA dissection was strongly influenced by the large use, until the recent past, of retrograde femoral perfusion.

The possibility of classifying the hemodynamic consequences of extracranial arterial stenoses to detect patients with failing cerebral perfusion and to appraise the presence of microembolic discharge illustrate the crucial role of TCD examination in the management and clinical follow-up of these patients, enabling us to tailor treatment to individual problems and to evaluate the results.

In patients with aortic dissection, chronic treatment with antihypertensive agents is almost universally recommended to minimize progression of their disease.

As pointed out by Griepp and colleagues, ${ }^{20}$ because such treatment is often effective, hypertension has only intermittently emerged as a risk factor for rupture in recent studies.

On the other hand, hypertension is the most powerful, prevalent, and treatable risk factor for stroke. meta-analyses of randomized trials found that an average reduction in diastolic blood pressure of $6 \mathrm{~mm} \mathrm{Hg}$ produces a $42 \%$ reduction in stroke incidence. ${ }^{21,22}$

In our study uncontrolled hypertension was found to be an independent predictor of neurologic events in the entire cohort of patients (BA residual dissection and control groups).

Resistant hypertension ${ }^{14}$ was quite a common finding in the presence of BA dissection and represented a major therapeutic problem in $9(21.4 \%)$ of 42 subjects. All affected subjects presented with important modifications of intracranial circulation caused by extracranial vessel flow obstruction. In none could secondary hypertension be demonstrated. We wondered whether a neurogenic mechanism could be advocated, given that all these subjects experi- 
enced an exacerbation of their hypertensive state after dissection.

Still, with regard to pressure control, one of the more serious problems during follow-up was the conviction of some referring physicians that in the presence of residual dissection, especially if extracranial vessel flow obstruction was demonstrated, a nonaggressive treatment of hypertension should be pursued.

Although definite guidelines of blood pressure control in the presence of extracranial carotid disease have not been established, ${ }^{23,24}$ we found the use TCD ultrasonography to be of great value in the evaluation of the effects of an antihypertensive medication on cerebral blood flow velocity, and consequently, we can provide these patients with an adequate antihypertensive prophylaxis against false channel evolution.

\section{Conclusions}

Residual dissection of the BAs exposes the patient to an increased risk of neurologic events in the territories dependent on the dissected arteries. Strict follow-up and identification of subjects at risk implies the exact knowledge of vessel anatomy and perfusion status. In this, perspective TCD examination plays an important role in the clinical work-up of these patients and enables the physician to tailor treatment to individual problems.

We thank Mr Massimo Gistri del Nicchio for his help with the Doppler studies. We would also like to thank all the patients who participated in the study.

\section{References}

1. Zurbrugg HR, Leupi F, Schupbach P, Althaus U. Duplex scanner study of carotid artery dissection following surgical treatment of aortic dissection type A. Stroke. 1988;19:970-6.

2. Zielinski T, Wolkanin-Bartnik J, Janaszek-Sitkowska H, Biederman A, Rynkun D, Makowiecka-Ciesla M, et al. Persistent dissection of carotid artery in patients operated on for type A acute aortic dissection-carotid ultrasound follow-up. Int J Cardiol. 1999;70:133-9.

3. Sturzenegger M, Mattle HP, Rivoir A, Baumgartner RW. Ultrasound findings in carotid artery dissection: analysis of 43 patients. Neurology. 1995;45:691-8.

4. Bartels E. Vertebral sonography. In: Tagler CH, Babikian VL, Gomez CR, editors. Neurosonology. St Louis: Mosby; 1996. p. 83-100.

5. Aaslid R, Markwalder TM, Nornes H. Non-invasive transcranial Doppler ultrasound recording of flow velocity in basal cerebral arteries. J Neurosurg. 1982;57:769-74.
6. Silvestrini M, Vernieri F, Pasqualetti P, Matteis M, Passarelli F, Troisi E, et al. Impaired cerebral vasoreactivity and risk of stroke in patients with asymptomatic carotid artery stenosis. JAMA. 2000;283:2122-7.

7. Markus HS, Harrison MJ. Estimation of cerebrovascular reactivity using transcranial Doppler, including the use of breath-holding as the vasodilatatory stimulus. Stroke. 1992;23:668-73.

8. Apruzzese A, Silvestrini M, Floris R, Vernieri F, Bozzao A, Hagberg $\mathrm{G}$, et al. Cerebral hemodynamics in asymptomatic patients with internal carotid artery occlusion: a dynamic susceptibility contrast MR and transcranial Doppler study. AJNR Am J Neuroradiol. 2001;22:1062-7.

9. Consensus Committee of the Ninth International Cerebral Hemodynamics Symposium. Basic identification criteria of Doppler microembolic signals. Stroke. 1995;26:1123.

10. Gardner DJ, Gosink BB, Kallman CE. Internal carotid artery dissections: Duplex ultrasound imaging. J Ultrasound Med. 1991;10:607-14.

11. Vernieri F, Pasqualetti P, Passarelli F, Rossini PM, Silvestrini M. Outcome of carotid artery occlusion is predicted by cerebrovascular reactivity. Stroke. 1999;30:593-8.

12. Inhaka R, Gentleman R. R: a language for data analysis and graphics. J Comput Graphical Stat. 1996;5:299-314.

13. Ayad M, Vinuela F, Rubinstein EH. The suboccipital carrefour: cervical and vertebral arterial anastomosis. AJNR Am J Neuroradiol. 1998; 19:925-31.

14. The Joint National Committee on Prevention, Detection, Evaluation, and Treatment of High Blood Pressure. The Sixth Report of the Joint National Committee on Prevention, Detection, Evaluation, and Treatment of High Blood Pressure. Arch Intern Med. 1997;157:2413-46.

15. Srinivasan J, Newell DW, Sturzenegger M, Mayberg MR, Winn HR. Transcranial Doppler in the evaluation of internal carotid artery dissection. Stroke. 1996;27:1226-30.

16. Baumgartner RW, Frick A, Kremer C, Oechslin E, Russi E, Turina J, et al. Microembolic signal counts increase during hyperbaric exposure in patients with prosthetic heart valves. J Thorac Cardiovasc Surg. 2001;122:1142-6.

17. Ergin MA, Philips RA, Galla JD, Lansman SL, Mendelson DS, Quintana CS, et al. Significance of distal false lumen after type A dissection repair. Ann Thorac Surg. 1994;57:820-5.

18. David TE, Armstrong S, Ivanov J, Barnard S. Surgery for acute type A aortic dissection. Ann Thorac Surg. 1999;67:1999-2001.

19. Van Arsdell GS, David TE, Butany J. Autopsies in acute type A aortic dissection. Circulation. 1998;98(suppl II):II299-303.

20. Griepp RB, Ergin MA, Galla JD, Lansman SL, McCullough JN, Nguyen KH, et al. Natural history of descending thoracic and thoracoabdominal aneurysms. Ann Thorac Surg. 1999;67:1927-30; discussion 1953-8.

21. MacMahon S, Rodgers A. Blood pressure, antihypertensive treatment and stroke risk. J Hypertens Suppl. 1994;12:S5-14.

22. Collins R, Peto R, MacMahon S, Hebert P, Fiebach NH, Eberlein KA, et al. Blood pressure, stroke, and coronary heart disease, part 2: short-term reductions in blood pressure. Overview of randomised drug trials in their epidemiological context. Lancet. 1990;335:827-38.

23. The INDANA (Individual Data Analysis of Antihypertensive intervention trials) Project Collaborators. Effect of antihypertensive treatment in patients having already suffered a stroke. Stroke. 1997;28: 2557-62.

24. Gubitz G, Sandercock P. Prevention of ischaemic stroke. BMJ. 2000; 321:1455-9. 